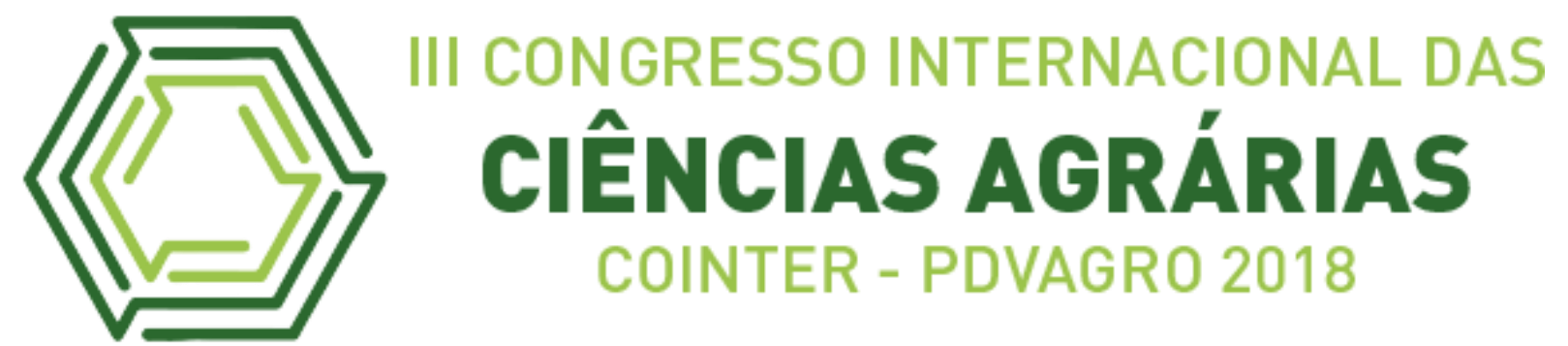

\title{
CARACTERIZAÇÃ̃ QUÍMICA DO SOLO EM BARRAGEM SUBTERRÂNEA NO SEMIÁRIDO PARAIBANO
}

\section{CHEMICAL CHARACTERIZATION OF SOIL IN UNDERGROUND DAM IN SEMIARID PARAIBANO}

Apresentação: Pôster

\section{Roseli Freire de Melo; Anderson Ramos de Oliveira² ; José Barbosa dos Anjos; Ítalo Luis Oliveira Santana ${ }^{4}$; Welson Lima Simoes ${ }^{5}$}

\section{DOI: https://doi.org/10.31692/2526-7701.IIICOINTERPDVAGRO.2018.00377}

\section{Introdução}

É notória a importância das barragens subterrâneas como tecnologia de captação e armazenamento de água de chuva para a produção de alimentos no Semiárido. Estudos realizados pela Embrapa Semiárido, em parceria com outras instituições, têm ressaltado, com destaque, a barragem subterrânea como alternativa viável para captação e armazenamento de água para produção de alimentos (BRITO, 1989; MELO et al., 2013). Entretanto, tem sido observado que as barragens subterrâneas que têm um camalhão em sua parede com nível acima do solo favorecem o acúmulo de sedimentos na área de captação, podendo resultar na melhoria da fertilidade do solo. No entanto, fazse necessário o monitoramento do solo para acompanhar a evolução de sua fertilidade na área de captação/plantio. Por ser um ambiente sedimentar, numa barragem subterrânea, pode-se ter variação nas características químicas do solo em profundidade. Por esse motivo, justificam-se estudos nesses ambientes que permitam conhecer a fertilidade do solo até camadas mais profundas. Consequentemente, esse estudo pode favorecer o planejamento da adubação a ser aplicada no plantio ou em cobertura.

As principais vantagens da barragem subterrânea são a acumulação de água com reduzida perda por evapotranspiração, comparativamente com os reservatórios de acumulação de água superficial, cujas perdas podem alcançar até $2000 \mathrm{~mm}$ por ano (MOURA et al., 2007); os menores riscos de salinização e a não ocupação de áreas agricultáveis (BRITO et al., 1989). Além disso, a

\footnotetext{
${ }^{1}$ Pesquisadora, Eng. Agrônoma, D.Sc., Embrapa Semiárido, Petrolina, PE, e-mail, roseli.melo@ embrapa.br

${ }^{2}$ Pesquisador, Eng. Agrônomo, D.Sc.r., Embrapa Semiárido, Petrolina, PE, anderson.oliveira@embrapa.br

${ }^{3}$ Pesquisador, Eng. Agrônomo, M.Sc., Embrapa Semiárido, Petrolina, PE, jose-barbosa.anjos@embrapa.br

${ }^{4}$ Estudante do Curso de Biologia da Universidade de Pernambuco, olivveira09@gmail.com

${ }^{5}$ Pesquisador, Eng. Agrônomo, D.Sc.r., Embrapa Semiárido, Petrolina, PE, welson.simoes@embrapa.br
} 
agregação de práticas de manejo, como a adubação orgânica, garante maior permanência da água no solo. É possível, ainda, que a barragem subterrânea funcione como uma tecnologia de conservação ambiental, evitando perdas de solos e sedimentos. Neste contexto, o presente trabalho teve como objetivo avaliar as características químicas do solo em diferentes extratos (início, meio e fim) e profundidade na área de captação/plantio em uma barragem subterrânea, localizada em Arara, PB.

\section{Fundamentação Teórica}

A barragem subterrânea é uma tecnologia que consiste na construção de uma parede impermeável (septo), no sentido transversal à descida das águas, que impede o movimento natural descendente de água e do perfil do solo em uma linha de drenagem, rio ou riacho, promovendo, assim, seu armazenamento no espaço poroso do solo, para que possa ser utilizada para cultivos "in situ" ou para irrigar áreas "ex situ” ou para a dessedentação dos animais (ONDER; YILMAZ, 2005; SILVA et al., 2007). Os resultados apontam que, após a implantação da barragem subterrânea, os agricultores familiares têm conseguido, além da produção de alimentos e forragem para a criação animal, a recuperação da área pelo acúmulo de sedimentos (DIAS et al., 2014). Desta forma, comprova-se que a barragem subterrânea proporciona soberania e segurança alimentar e nutricional às famílias e ainda favorece a conservação ambiental.

A alteração nas características químicas em barragem subterrânea tem relação com os barramentos feitos nas linhas de drenagem, por meio da construção dos septos, para retenção dos fluxos que trazem sedimentos que são depositados no espaço de acomodação proporcionado pelo barramento (NICHOLS, 2009).

É evidente que os sedimentos advindos dos fluxos descendentes trazem nutrientes. Estes podem se acumular devido ao barramento, levando os solos da barragem a apresentarem elevada saturação de bases, além da alteração em outras características químicas (NASCIMENTO et al., 2015). De acordo com Lucena et al. (2015), as características químicas podem ter influência positiva decorrente do acúmulo de matéria orgânica, influenciando diretamente a fertilidade do solo, mas merece atenção quanto à acumulação de sais. O tipo de solo, o relevo e os sistemas de manejo são fatores que devem ser analisados ao avaliar e monitorar a influência das barragens na qualidade dos solos.

\section{Metodologia}


O estudo foi realizado em uma barragem subterrânea, localizada no Município de Arara Semiárido da Paraíba, no ano de 2016, aproximadamente sete anos após sua construção. Foram coletadas amostras de solo compostas nas áreas de captação/plantio que foi dividida em três extratos: início (área mais próxima do sangradouro), meio (aproximadamente no meio da barragem) e fim (considerada a parte mais distante do sangradouro). Também, foi feita coleta na área de referência, à jusante da barragem subterrânea, conforme ilustrado na Figura 1. O delineamento experimental adotado foi em blocos casualizados, utilizando-se um esquema fatorial $4 \times 3$ (quatro ambientes e três profundidades) e quatro repetições As amostras compostas de solo foram coletadas no final do período chuvoso. As análises químicas foram realizadas no Laboratório de Solos da Embrapa Semiárido, de acordo com metodologias já descritas (EMBRAPA, 1997), sendo determinados, para os três extratos de amostragem: $\mathrm{pH}$, fósforo $(\mathrm{P})$, potássio $(\mathrm{K})$, cálcio $(\mathrm{Ca})$, magnésio $(\mathrm{Mg})$, alumínio $(\mathrm{Al})$, alumínio trocável $(\mathrm{H}+\mathrm{Al})$, sódio $(\mathrm{Na})$, soma de bases $(\mathrm{S})$, capacidade de troca de cátions (CTC), saturação de bases (V), condutividade elétrica (CE), relação de adsorção de sódio (RAS) e Carbono Total (CTotal) e os micronutrientes cobre $(\mathrm{Cu})$ e ferro $(\mathrm{Fe})$. Estes resultados foram submetidos à análise de variância (ANOVA) e as médias foram comparadas aplicandose o teste de Tukey a $5 \%$ de probabilidade.

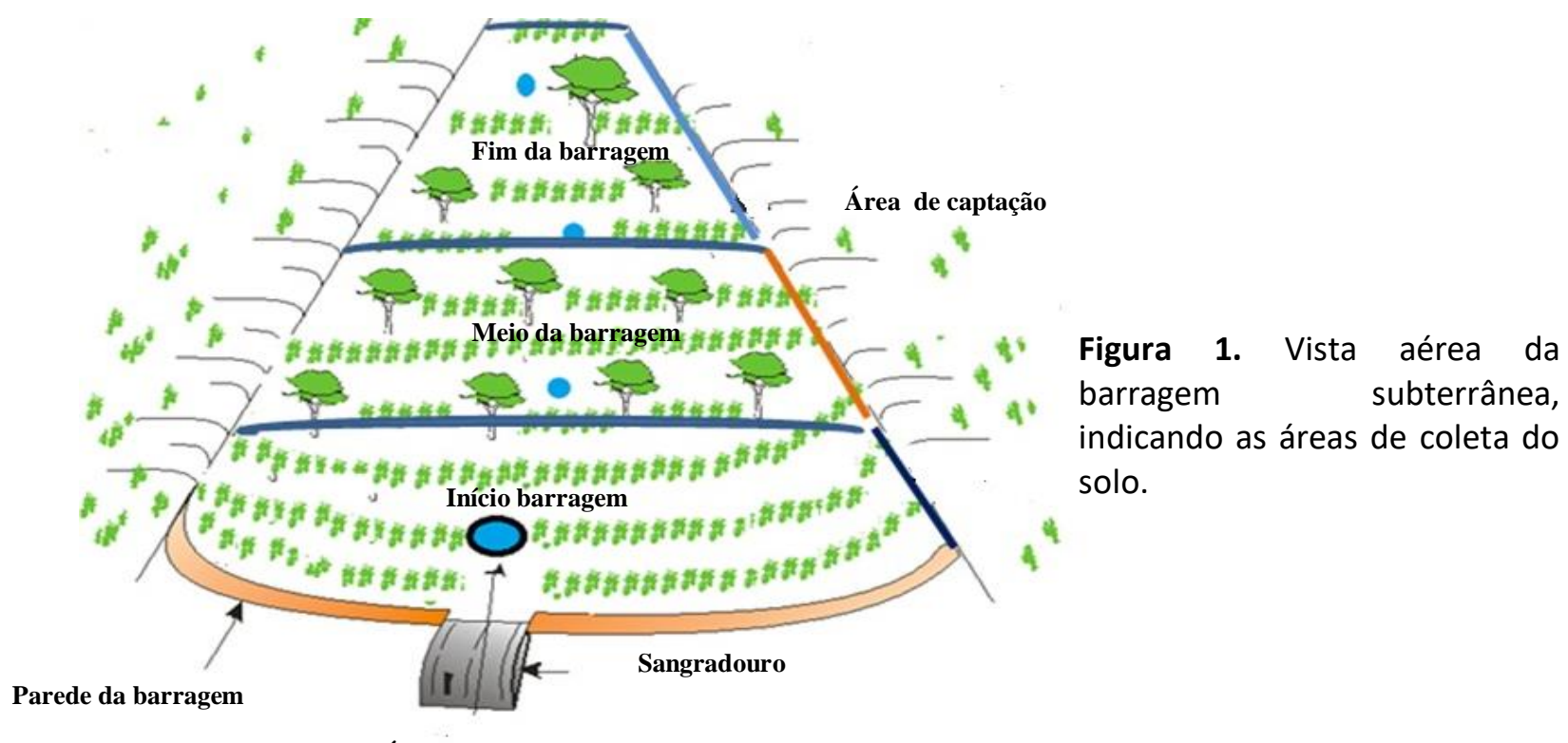

Área de referência

\section{Resultados e Discussão}


De acordo com os resultados, é possível observar, na Tabela 1, que os maiores valores de carbono estão nas camadas de $0-20$ e de $20-40 \mathrm{~cm}(\mathrm{P}>0,05)$, reduzindo na profundidade de 40-60 cm em relação à primeira camada, nos três extratos de amostragem (início, meio e fim da barragem). Além disso, é notório o aumento nos valores do Ctotal e do $\mathrm{P}$ na barragem subterrânea em relação à área de referência. Os valores encontrados para as variáveis $\mathrm{pH}, \mathrm{K}$, $\mathrm{Na}, \mathrm{Mg}, \mathrm{Al}, \mathrm{Cu}$ e $\mathrm{Fe}$ não apresentaram diferenças estatísticas em profundidade, em nenhum dos extratos amostrados. No entanto, os valores de $\mathrm{P}$ variaram estatisticamente em profundidade, sendo que, no início da barragem, os maiores valores foram encontrados nas profundidades de $20-40 \mathrm{~cm}\left(9,56 \mathrm{mg} \cdot \mathrm{dm}^{-3}\right)$ e de $40-60 \mathrm{~cm}\left(8,80 \mathrm{mg} \cdot \mathrm{dm}^{-3}\right)$, diferindo estatisticamente do valor encontrado na camada de 0-20 $\mathrm{cm}\left(6,37 \mathrm{mg}_{\text {.dm }}{ }^{-3}\right)$. Já no meio da barragem, os valores de $\mathrm{P}$ foram mais elevados, com diferença estatística para a maior profundidade, apresentando, nas camadas de 0-20; 20-40 e 40-60 cm, os valores de 42,95 mg.dm ${ }^{-3}, 37,28 \mathrm{mg} \cdot \mathrm{dm}^{-3}$ e 9,36 mg.dm ${ }^{-3}$, respectivamente. Os resultados de $\mathrm{Ca}$ e $\mathrm{H}+\mathrm{Al}$ diferiram apenas nas amostras coletadas no meio da barragem, com maiores valores na camada superficial, o que refletiu em maior valor de CTC do solo.

\begin{tabular}{|c|c|c|c|c|c|c|c|c|c|c|c|c|c|c|c|}
\hline \multirow[t]{3}{*}{ Ambientes } & \multicolumn{15}{|c|}{ Característica química } \\
\hline & $\mathrm{CE}$ & $\mathrm{C}$ total & $\mathrm{pH}$ & $\mathrm{P}$ & $\mathrm{K}$ & $\mathrm{Na}$ & $\mathrm{Ca}$ & $\mathrm{Mg}$ & $\mathrm{Al}$ & $\mathrm{H}+\mathrm{Al}$ & SB & CTC & $\mathrm{V}$ & $\mathrm{Cu}$ & $\mathrm{Fe}$ \\
\hline & $\mathrm{mS} \mathrm{cm}^{-1}$ & g. $\mathrm{kg}^{-1}$ & - & \multicolumn{9}{|c|}{ 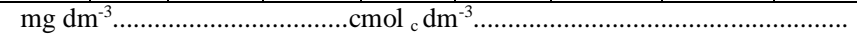 } & \multicolumn{3}{|c|}{$\% \quad$ \%...mg dm ${ }^{-3} \ldots \ldots \ldots$} \\
\hline \multicolumn{16}{|c|}{ INÍCIO DA BARRAGEM } \\
\hline $0-20$ & $0,13 \mathrm{a}$ & $4,20 \mathrm{a}$ & $5,47 \mathrm{a}$ & $6,37 b$ & $0,13 \mathrm{a}$ & $0,15 \mathrm{a}$ & $0,77 \mathrm{a}$ & $1,83 \mathrm{a}$ & $0,52 \mathrm{a}$ & $2,47 \mathrm{a}$ & $2,87 \mathrm{ab}$ & $5,33 \mathrm{a}$ & $54,73 a$ & $2,47 \mathrm{a}$ & $1281,53 a$ \\
\hline $20-40$ & $0,14 \mathrm{a}$ & $3,32 \mathrm{ab}$ & $5,57 \mathrm{a}$ & $9,56 \mathrm{a}$ & $0,11 \mathrm{a}$ & $0,27 \mathrm{a}$ & $0,70 \mathrm{a}$ & $2,23 \mathrm{a}$ & $0,78 \mathrm{a}$ & $2,97 \mathrm{a}$ & $3,30 \mathrm{a}$ & $6,30 \mathrm{a}$ & $54,93 \mathrm{a}$ & $2,42 \mathrm{a}$ & $1081,50 \mathrm{a}$ \\
\hline $40-60$ & $0,28 b$ & $2,21 b$ & $5,80 \mathrm{a}$ & $8,80 \mathrm{ab}$ & $0,11 \mathrm{a}$ & $0,19 \mathrm{a}$ & $1,23 \mathrm{a}$ & $2,23 \mathrm{a}$ & $0,12 \mathrm{a}$ & $2,17 \mathrm{a}$ & $3,77 \mathrm{~b}$ & $5,93 \mathrm{a}$ & $65,30 \mathrm{a}$ & $2,18 \mathrm{a}$ & $1616,50 \mathrm{a}$ \\
\hline \multicolumn{16}{|c|}{ MEIO DA BARRAGEM } \\
\hline $0-20$ & $0,21 \mathrm{a}$ & $2,56 a$ & $4,93 a$ & $42,95 a$ & $0,26 \mathrm{a}$ & $0,13 \mathrm{a}$ & $1,53 \mathrm{a}$ & $2,23 \mathrm{a}$ & $0,60 \mathrm{a}$ & $4,03 a$ & $4,17 \mathrm{a}$ & $8,20 \mathrm{a}$ & $50,87 \mathrm{a}$ & $1,82 \mathrm{a}$ & $753,67 \mathrm{a}$ \\
\hline $20-40$ & $0,23 \mathrm{a}$ & $1,33 a b$ & $4,97 \mathrm{a}$ & $37,28 \mathrm{a}$ & $0,19 \mathrm{a}$ & $0,14 \mathrm{a}$ & $0,83 b$ & $1,90 \mathrm{a}$ & $0,72 \mathrm{a}$ & $2,70 \mathrm{ab}$ & $3,10 \mathrm{~b}$ & $5,73 \mathrm{~b}$ & $53,97 \mathrm{a}$ & $1,45 \mathrm{a}$ & $1091,30 \mathrm{a}$ \\
\hline $40-60$ & $0,23 \mathrm{a}$ & $0,71 b$ & $5,20 \mathrm{a}$ & $9,36 \mathrm{~b}$ & $0,19 \mathrm{a}$ & $0,11 \mathrm{a}$ & $0,73 b$ & $1,90 \mathrm{a}$ & $0,53 \mathrm{a}$ & $2,40 \mathrm{~b}$ & $2,93 b$ & $5,30 \mathrm{~b}$ & $55,20 \mathrm{a}$ & $1,34 \mathrm{a}$ & $895,63 a$ \\
\hline \multicolumn{16}{|c|}{ FIM DA BARRAGEM } \\
\hline $0-20$ & $0,19 \mathrm{ab}$ & $1,55 \mathrm{a}$ & $5,20 \mathrm{a}$ & $35,82 \mathrm{a}$ & $0,33 \mathrm{a}$ & $0,16 \mathrm{a}$ & $0,93 \mathrm{a}$ & $2,27 \mathrm{a}$ & $0,47 \mathrm{a}$ & $3,23 \mathrm{a}$ & $3,70 \mathrm{a}$ & $6,93 \mathrm{a}$ & $54,00 \mathrm{a}$ & $1,34 \mathrm{a}$ & $737,43 a$ \\
\hline $20-40$ & $0,23 \mathrm{ab}$ & $1,03 \mathrm{ab}$ & $5,13 \mathrm{a}$ & $11,60 \mathrm{~b}$ & $0,24 \mathrm{a}$ & $0,15 \mathrm{a}$ & $0,97 \mathrm{a}$ & $2,10 \mathrm{a}$ & $0,72 \mathrm{a}$ & $3,10 \mathrm{a}$ & $3,47 \mathrm{a}$ & $6,60 \mathrm{a}$ & $54,07 \mathrm{a}$ & $1,24 \mathrm{a}$ & $1065,77 \mathrm{a}$ \\
\hline $40-60$ & $0,29 a$ & $0,88 \mathrm{~b}$ & $5,30 \mathrm{a}$ & $8,57 \mathrm{~b}$ & $0,23 \mathrm{a}$ & $0,17 \mathrm{a}$ & $0,93 \mathrm{a}$ & $1,90 \mathrm{a}$ & $0,50 \mathrm{a}$ & $2,77 \mathrm{a}$ & $3,23 a$ & $6,00 \mathrm{a}$ & $55,43 a$ & $1,52 \mathrm{a}$ & $652,80 \mathrm{a}$ \\
\hline \multicolumn{16}{|c|}{ ÁREA DE REFERÊNCIA } \\
\hline $0-20$ & $0,12 \mathrm{a}$ & $0,96 \mathrm{a}$ & $5,13 \mathrm{a}$ & $0,54 \mathrm{a}$ & $0,16 \mathrm{a}$ & $0,16 \mathrm{a}$ & $0,70 \mathrm{a}$ & $2,90 \mathrm{a}$ & $0,94 \mathrm{a}$ & $4,60 \mathrm{a}$ & $4,00 \mathrm{a}$ & $8,77 \mathrm{a}$ & $46,70 \mathrm{a}$ & $2,47 \mathrm{a}$ & $201,50 \mathrm{a}$ \\
\hline $20-40$ & $0,12 \mathrm{a}$ & $0,70 \mathrm{a}$ & $5,17 \mathrm{a}$ & $0,52 \mathrm{a}$ & $0,13 \mathrm{a}$ & $0,14 \mathrm{a}$ & $0,60 \mathrm{a}$ & $2,90 \mathrm{a}$ & $0,93 \mathrm{a}$ & $4,10 \mathrm{a}$ & $3,80 \mathrm{a}$ & $8,23 a$ & $42,77 \mathrm{a}$ & $2,32 \mathrm{a}$ & $199,03 a$ \\
\hline $40-60$ & $0,12 \mathrm{a}$ & $0,67 \mathrm{a}$ & $5,17 \mathrm{a}$ & $0,50 \mathrm{a}$ & $0,12 \mathrm{a}$ & $0,12 \mathrm{a}$ & $0,53 \mathrm{a}$ & $2,90 \mathrm{a}$ & $0,90 \mathrm{a}$ & $4,00 \mathrm{a}$ & $3,60 \mathrm{a}$ & $8,17 \mathrm{a}$ & $42,10 \mathrm{a}$ & $2,25 \mathrm{a}$ & $197,70 \mathrm{a}$ \\
\hline
\end{tabular}

Tabela 1. Características químicas das amostras de solo coletadas em diferentes profundidades, na época do inverno no ano de 2016 (mês de maio), em barragem subterrânea, localizada no Município de Arara, no Estado 
da Paraíba. Fonte própria

As características químicas nos diferentes extratos de amostragem variaram, inclusive, com relação aos valores de carbono orgânico. Esses valores podem ser atribuídos, segundo Salcedo e Sampaio (2008), “à variabilidade espacial e temporal na disponibilidade hídrica da região semiárida que exerce o principal controle na produção de biomassa e nos aportes de resíduos vegetais no solo". Entende-se, dessa forma, que o material orgânico aportado nos solos de barragem subterrânea tende a se acumular mais no ambiente próximo ao sangradouro, por causa das características de relevo do solo (6\% de declividade), conforme citado por Lucena et al. (2015). Vale ressaltar, também, que a decomposição desse material em um curto período de tempo pode ter refletido o baixo acúmulo de carbono orgânico em profundidade. Porém, na área próxima ao sangradouro, de modo geral, a fertilidade do solo apresentou ganho em profundidade. Isto comprova que o acúmulo de sedimento em barragem subterrânea tende a melhorar a fertilidade do solo como mencionado por Dias et al. (2014).

\section{Conclusões}

Observou-se que a barragem subterrânea contribuiu para uma mudança positiva nas características químicas do solo em profundidade. As principais alterações nos valores de carbono orgânico foram observadas na área próxima ao sangradouro e na camada superficial.

\section{Referências}

BRITO, L. T. de L.; SILVA, A. de S.; MACIEL, J. L.; MONTEIRO, M. A. R. Barragem subterrânea I: construção e manejo. Petrolina: EMBRAPA-CPATSA, 1989. 38 p. il. (EMBRAPA CPATSA. Boletim de Pesquisa, 36).

DIAS, I.; FAÉ, V.; SILVA, M. S. L. da. Relatório de avaliação dos impactos das tecnologias geradas pela Embrapa. Rio de Janeiro: Embrapa Solos, 2014. 34p.

EMBRAPA - Empresa Brasileira de Pesquisa Agropecuária. Centro Nacional de Pesquisa de Solos, Manual de Métodos de Análise de Solos. 2. ed. Rio de Janeiro, Embrapa, 1997. 212.p.

LUCENA, W. B.; FERREIRA, G. B., SILVA, M. S. L.; MOREIRA, M. M.; SILVA, M. J. S.; SOUZA, M. S. Avaliação de atributos químicos em solos com barragem subterrânea em agroecossistemas do semiárido. In: CONGRESSO BRASILEIRO DE CIÊNCIA DO SOLO, 35., 2015, Natal. O solo e suas múltiplas funções: anais. Natal: Sociedade Brasileira de Ciência do Solo, 2015. 
MELO, R. F. de; ANJOS, J. B. dos; SILVA, M. S. L. da; PEREIRA, L. A.; BRITO, L. T. de L. Barragem subterrânea: tecnologia para armazenamento de água e produção de alimentos. Petrolina: Embrapa Semiárido, 2013. (Embrapa Semiárido. Circular Técnica, 104). Disponível em: Acesso em: 24 de junho de 2018.

MOURA, M. S. B. de; GALVINCIO, J. D.; BRITO, L. T. de L.; SOUZA, L. S. B. DE; SÁ, I. I. S.; SILVA, T. G. F. da. Potencialidades de água da chuva no Semi-Árido brasileiro. Petrolina: Embrapa Semiárido, 2007.

NASCIMENTO, A. F.; SILVA, M. S. L.; MARQUES, F. A.; OLIVEIRA NETO, M. B.; PARAHYBA, R. B. V.; AMARAL, A. J. Caracterização geoambiental em áreas de barragens subterrâneas no Semiárido brasileiro / Alexandre Ferreira do Nascimento... [et al.]. - Dados eletrônicos. - Rio de Janeiro, RJ: Embrapa Solos; Recife, PE: Embrapa Solos UEP Recife, 2015.

NICHOLS, G. Sedimentology and Stratigraphy. 2nd ed. United Kingdom: Wiley Blackwell, 2009. 419 p.

ONDER, H.; YILMAZ, M. Underground dams: a tool of sustainable development and management of groundwater resources. European Water, Athens, v. 11-12, p. 35-45, 2005

SILVA, M. S. L da; MENDONÇA, A. E. S.; ANJOS, J. B.; HONÓRIO, A. P. M.; SILVA, A. S.; BRITO, L. T. L. Barragem subterrânea: água para produção de alimentos. In: BRITO, L. T. L.; MOURA, M. S. B.; GAMA, G. F. (Org.). Potencialidades da água de chuva no Semiárido brasileiro. Petrolina, PE: Embrapa Semi-Árido, 2007. p. 121-137. 